\title{
ELECTRON HOLOGRAPHY OF NANOSTRUCTURED MAGNETIC MATERIALS
}

\author{
R. E. DUNIN-BORKOWSKI ${ }^{\mathrm{a}, \mathrm{b}}$, B. KARDYNAL ${ }^{\mathrm{c}, \mathrm{d}}$, M. R. MCCARTNEY ${ }^{\mathrm{a}}$, \\ M. R. SCHEINFEIN ${ }^{\mathrm{e}, \mathrm{f}}$, DAVID J. SMITH ${ }^{\mathrm{a}, \mathrm{e}}$ \\ ${ }^{a}$ Center for Solid State Science, Arizona State University, Tempe, AZ 85287-1704 \\ b Now at: Department of Materials, University of Oxford, Parks Road, Oxford OX1 3PH, UK \\ c Center For Solid State Electronics Research, Arizona State University, Tempe, AZ 85287-6206 \\ ${ }^{d}$ Now at: Clarendon Laboratory, University of Oxford, Parks Road, Oxford OX1 3PU, UK \\ e Department of Physics and Astronomy, Arizona State University, Tempe, AZ 85287-1504 \\ f Now at: FEI, 7451 NW Evergreen Parkway, Hillsboro, OR 97124
}

\begin{abstract}
Off-axis electron holography and micromagnetic calculations that involve solutions to the Landau-Lifshitz-Gilbert equations are used to study magnetization reversal processes in lithographically patterned submicron-sized $\mathrm{Co}$ and $\mathrm{Co} / \mathrm{Au} / \mathrm{Ni}$ magnetic elements.
\end{abstract}

\section{INTRODUCTION}

A detailed understanding of magnetic nanostructures is essential for their utilization in information storage applications such as high density recording media and read heads. Such applications require reproducible magnetic domain structures and a good understanding of the interactions between neighboring elements. Here, we use off-axis electron holography in the transmission electron microscope (TEM) [1] to study the magnetic microstructure of submicronsized elements that have been patterned lithographically onto electron transparent $\mathrm{Si}_{3} \mathrm{~N}_{4}$ windows. We then compare our results with solutions to the Landau-Lifshitz-Gilbert equations [2]. The microscope geometry for off-axis electron holography and the approach used to obtain the phase of the holographic interference fringes, which is sensitive to the in-plane component of the magnetic induction integrated in the incident beam direction, are shown in Figs. 1a and 1b, respectively. Further details about the application of the technique can be found elsewhere [3].

Off-axis electron holograms were recorded at $200 \mathrm{kV}$ using a Philips CM200-FEG TEM equipped with a field-emission electron source, an electrostatic (rotatable) biprism located in the selected-area aperture plane and a $1024 \times 1024$ pixel Gatan 794 multi-scan CCD camera. An additional Lorentz minilens $\left(\mathrm{C}_{\mathrm{S}}=8 \mathrm{~m}\right.$ and $1.2 \mathrm{~nm}$ line resolution at $\left.200 \mathrm{kV}\right)$, located in the bore of the objective lens pole-piece, allowed images to be obtained with the main objective lens switched off and the sample located in almost field-free conditions. The objective lens could also be excited slightly and the sample tilted by up to $\pm 30^{\circ}$ in order to apply a known in-plane magnetic field, allowing magnetization processes to be followed in situ through hysteresis cycles. Reference holograms were acquired from the adjacent silicon nitride to remove artifacts caused by local irregularities of the image/recording system, and the mean inner potential contribution to the holographic phase was always subtracted to obtain the magnetic contribution of primary interest [3]. 


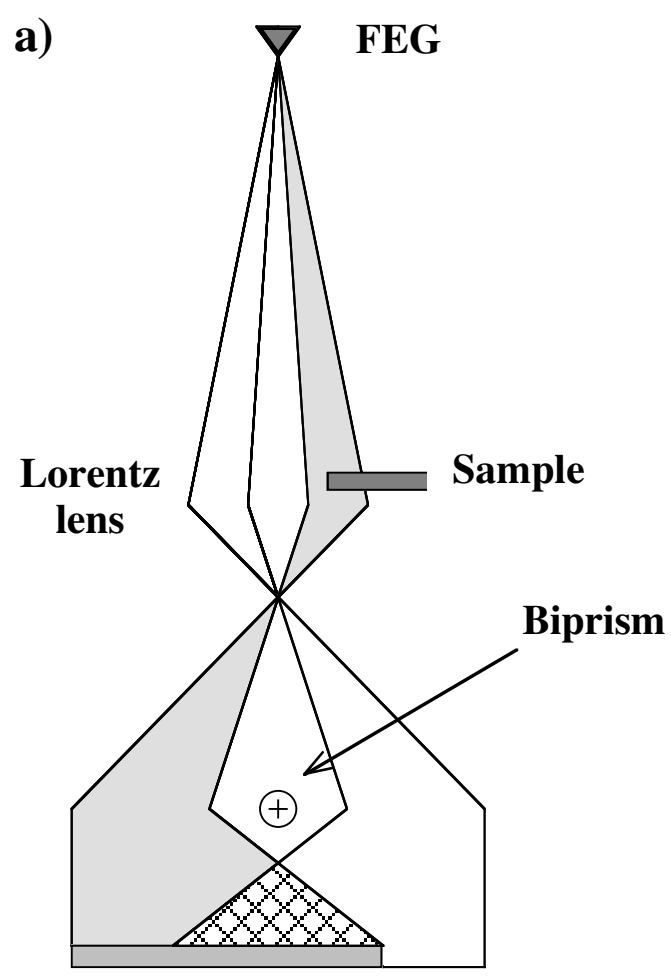

b)

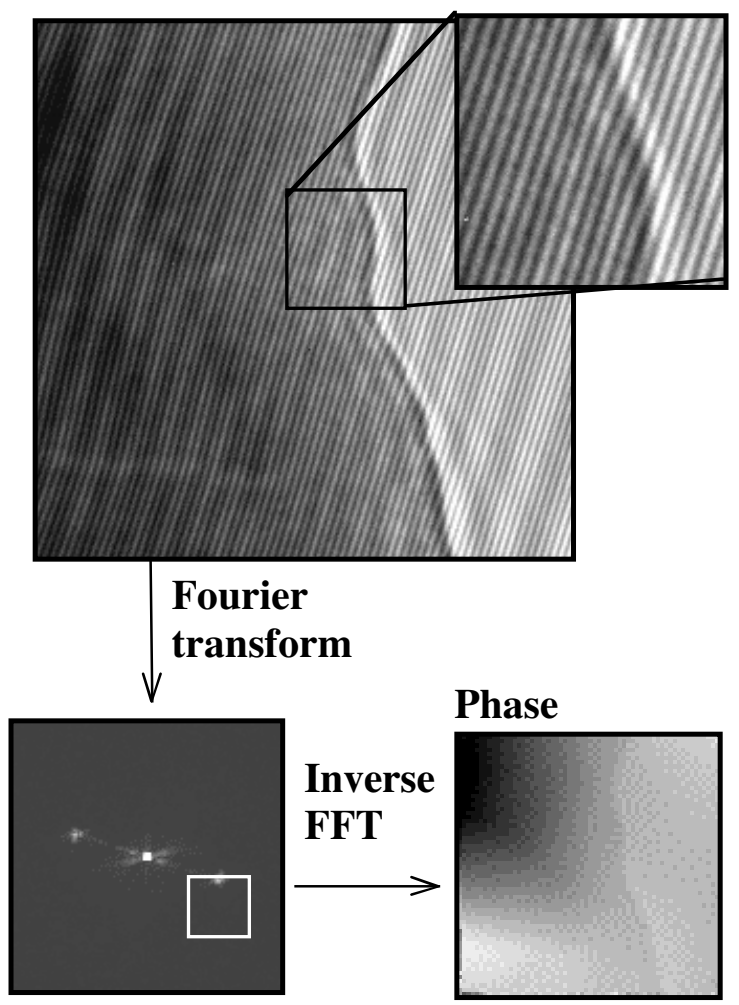

Fig.1. a) Setup used to generate off-axis electron holograms. Field emission source provides coherent illumination and electrostatic biprism causes overlap of object and reference waves. Lorentz lens allows imaging in close-to-field-free conditions. b) Off-axis electron hologram from thin crystal with enlargement showing interference fringes within sample. Fourier transform of hologram and phase obtained after inverse Fourier transform of sideband are also shown.

\section{RESULTS}

We begin by comparing holographic data from two 30nm-thick Co nanostructures with simulations. Figure 2a shows a representative hologram taken from one end of an ordered, close proximity (170nm) array of elements (Fig. 2b), whose intended cross-sectional geometry is given in Fig. 2c. The measured magnetic contribution to the holographic phase for a hysteresis loop is shown in Fig. $2 d$ for an in-plane field of between \pm 1930 Oe and an out-of-plane field of 3600 Oe. The holographic phase contours follow lines of constant magnetization, and their separation is proportional to the in-plane component of the magnetic induction integrated in the incident beam direction. Computed results are shown in Fig. 2e for initial conditions that were selected to best match the data [5]. The vortex helicities match those measured and almost all of the S-shaped domain structures are reproduced. However, the fact that differences in the starting state are of importance in the formation of subsequent domain structures is illustrated by Fig. $2 \mathrm{f}$, in which the vortex unrolls in the opposite sense in the second half of the cycle after changing the initial conditions slightly. Simulations also showed that the domain structures are sensitive to the outof-plane component of the applied field and to interactions between neighboring elements [5]. 
a)

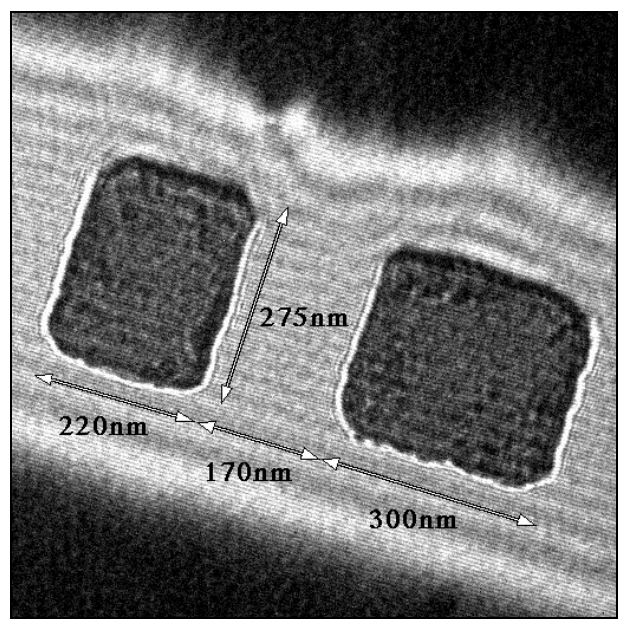

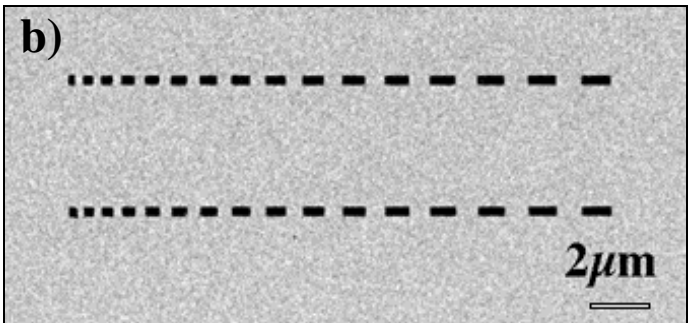

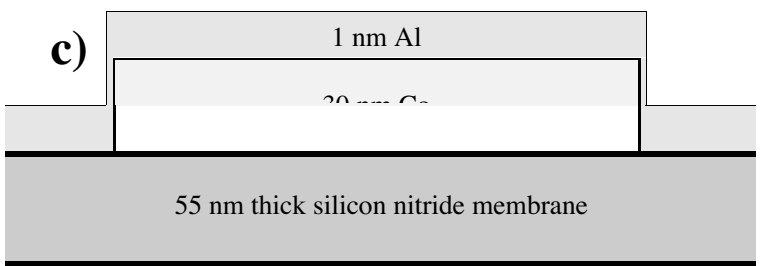

d)
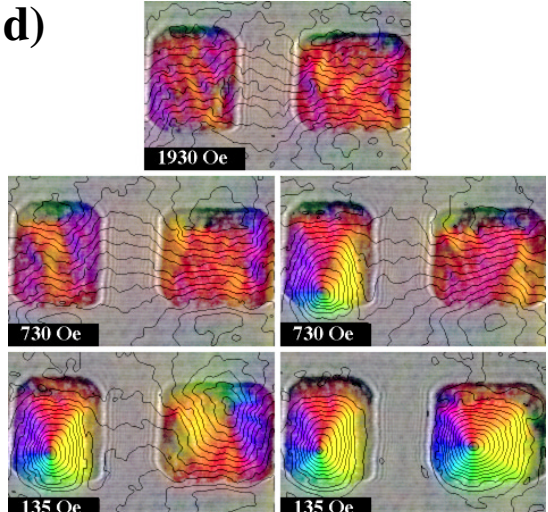

135 Oe

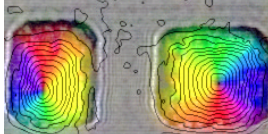

0 Oe
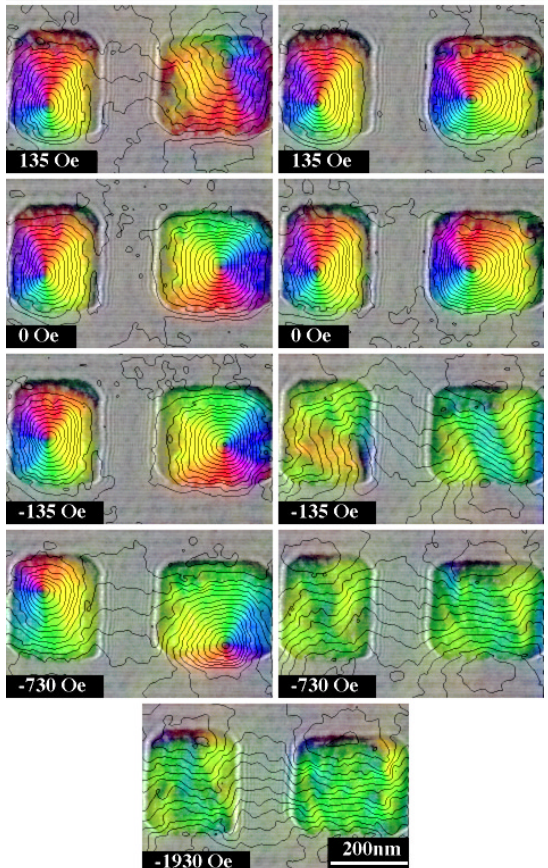

$135 \mathrm{Oe}$

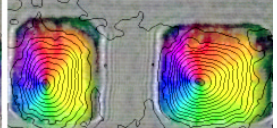

e)
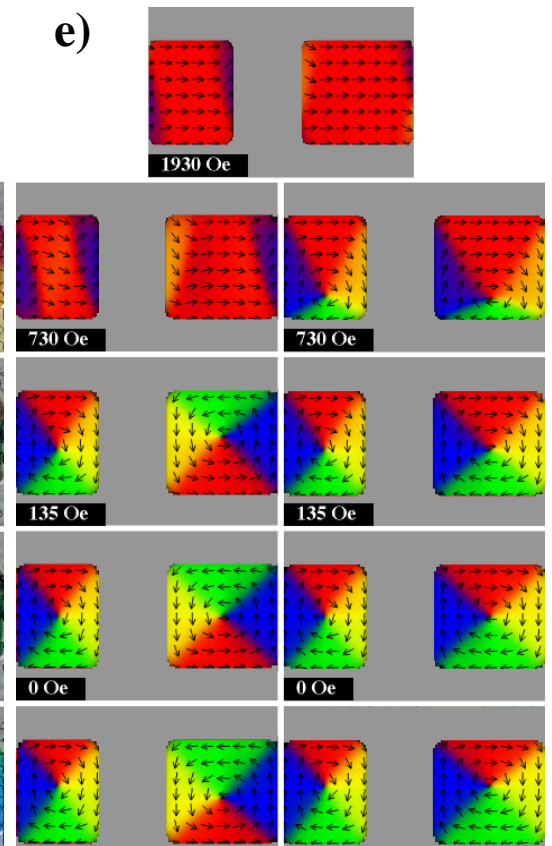

$-135 \mathrm{Oe}$

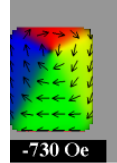

$-730 \mathrm{Oe}$
1930 Oe
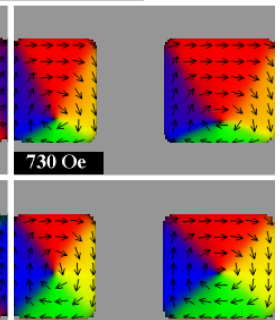

135 Oe
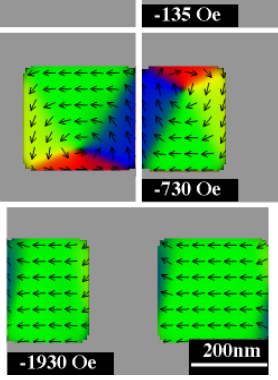

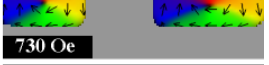

f)
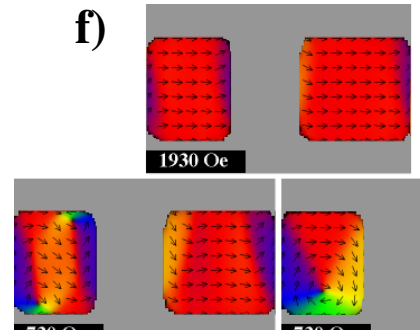

$730 \mathrm{Oe}$

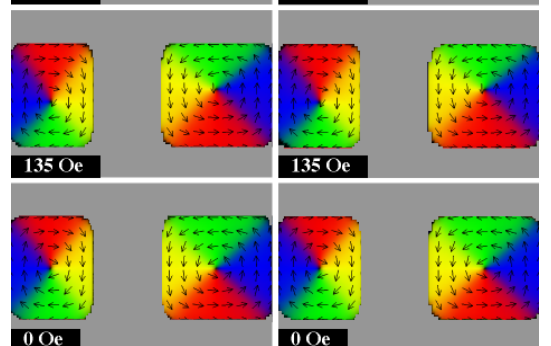

$0 \mathrm{Oe}$

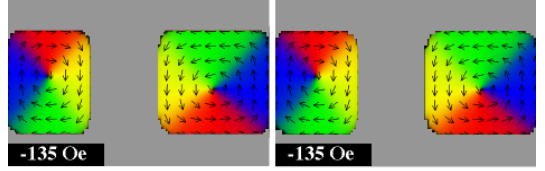

$-135 \mathrm{Oe}$
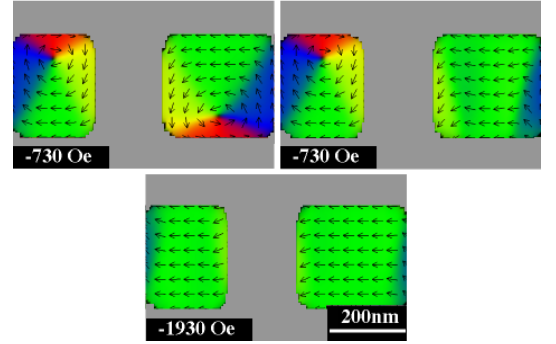

Fig.2. a) Off-axis electron hologram of $30 \mathrm{~nm}$ thick Co elements which form extreme left end of top linear array shown in b); c) Intended cross-sectional geometry of each element; d) Magnetic contributions to holographic phases during hysteresis cycle for 3600 Oe out-of-plane field and applied in-plane fields indicated. Phase contour spacing is $0.21 \pi$ radians; e) Simulation for initial S-state (large cell) and C-state (small cell) at start of cycle, and 3600 Oe field into page; f) As for e) but with very slight change to starting state and to curvature of corners of each element. 
Magnetic interactions between two thin, closely-separated ferromagnetic layers within individual lithographically defined spin valve or tunnel junction structures can also influence their switching mode and coercive field. Here, we examined $\mathrm{Co} / \mathrm{Au} / \mathrm{Ni}$ trilayers patterned into diamonds, ellipses and rectangular bars, which were separated laterally in order to reduce interelement interactions. Figure 3 a shows a hologram of two rectangular bars, which form the left end of the middle array shown in Fig. 3b. The intended cross-sectional geometry of each element is given in Fig. 3c. Results for the second largest element in each row are tabulated in three sets of four columns in Fig. 4. The left column in each set shows the magnetic contributions to the experimental holographic phases recorded during a complete hysteresis cycle. The switching fields of the diamond and elliptical shaped elements are smaller than for the rectangular bar, which never forms a vortex state and is also too narrow to form end-domains (which govern the reversal of larger rectangular elements). Instead, the phase contours curve at their ends by a maximum angle of $\sim 45^{\circ}$ just before magnetization reversal. The experimental phase contours have two distinct spacings in each element (narrower at higher applied fields and wider close to remanence). This observation provides direct evidence for the presence of ferromagnetic and antiferromagnetic coupling between the $\mathrm{Ni}$ and $\mathrm{Co}$, which can be understood with reference to the simulations shown in the remaining columns of Fig. 4. The columns labeled 'Co' and 'Ni' track the magnetization states of the individual magnetic layers within each element, while those labeled 'Total' show the computed holographic phase shifts, which can be compared directly with the experimental data. Changes in the 'Total' contour spacing are apparent between fields at which the Ni layer has reversed but the Co layer is still unchanged, and similar behavior is seen clearly in the form of steps in the experimental hysteresis cycles shown in Fig. 5, which are plotted in the form of the change in the magnetic contribution to the phase across each element.
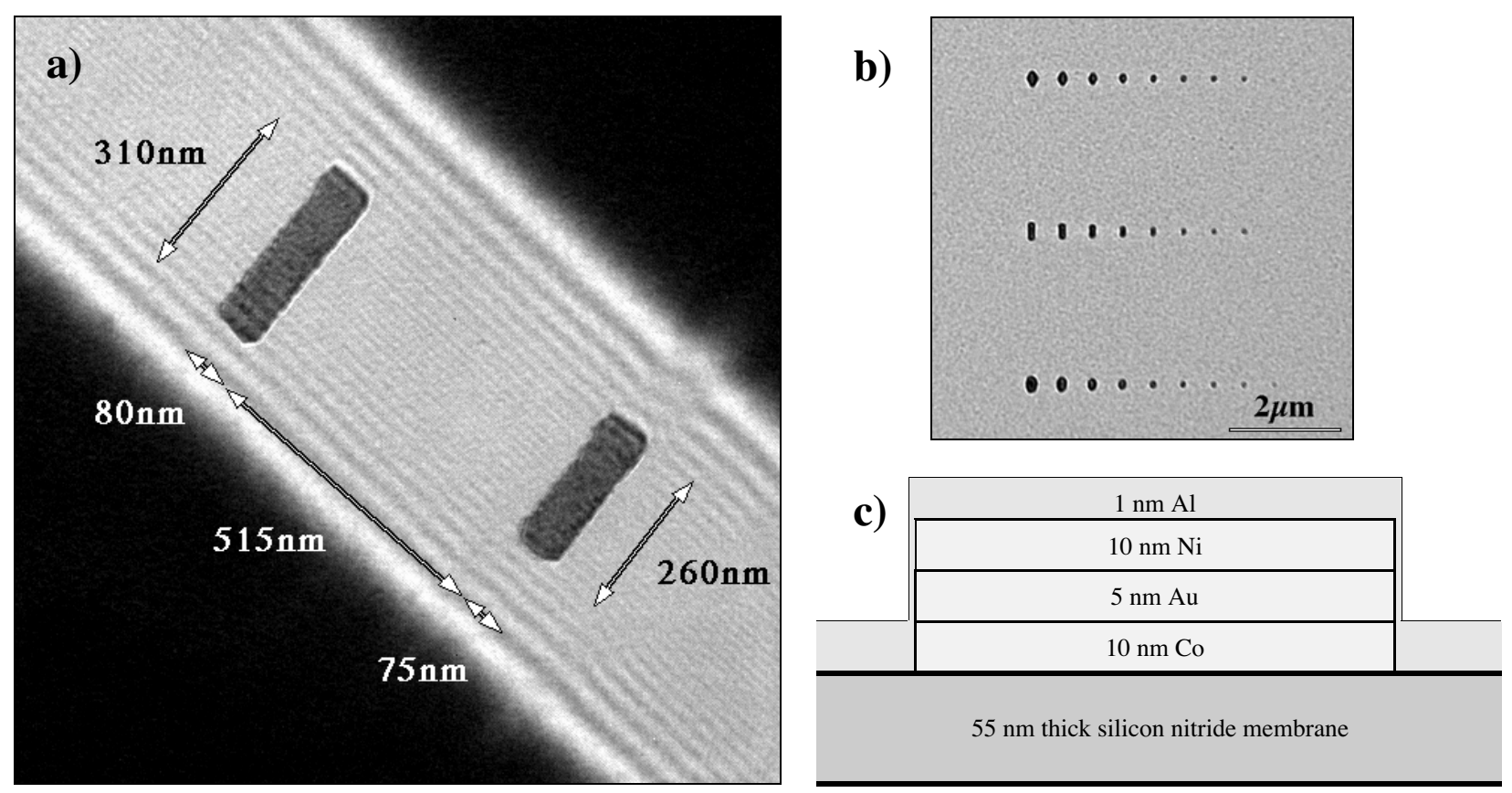

Fig.3. Off-axis electron hologram of two patterned bar-shaped elements of nominal layer sequence $10 \mathrm{~nm} \mathrm{Co} / 5 \mathrm{~nm} \mathrm{Au} / 10 \mathrm{~nm} \mathrm{Ni}$, also shown at left hand end of linear arrays of diamonds, bars and ellipses in b); c) Schematic diagram of intended cross-sectional geometry. 


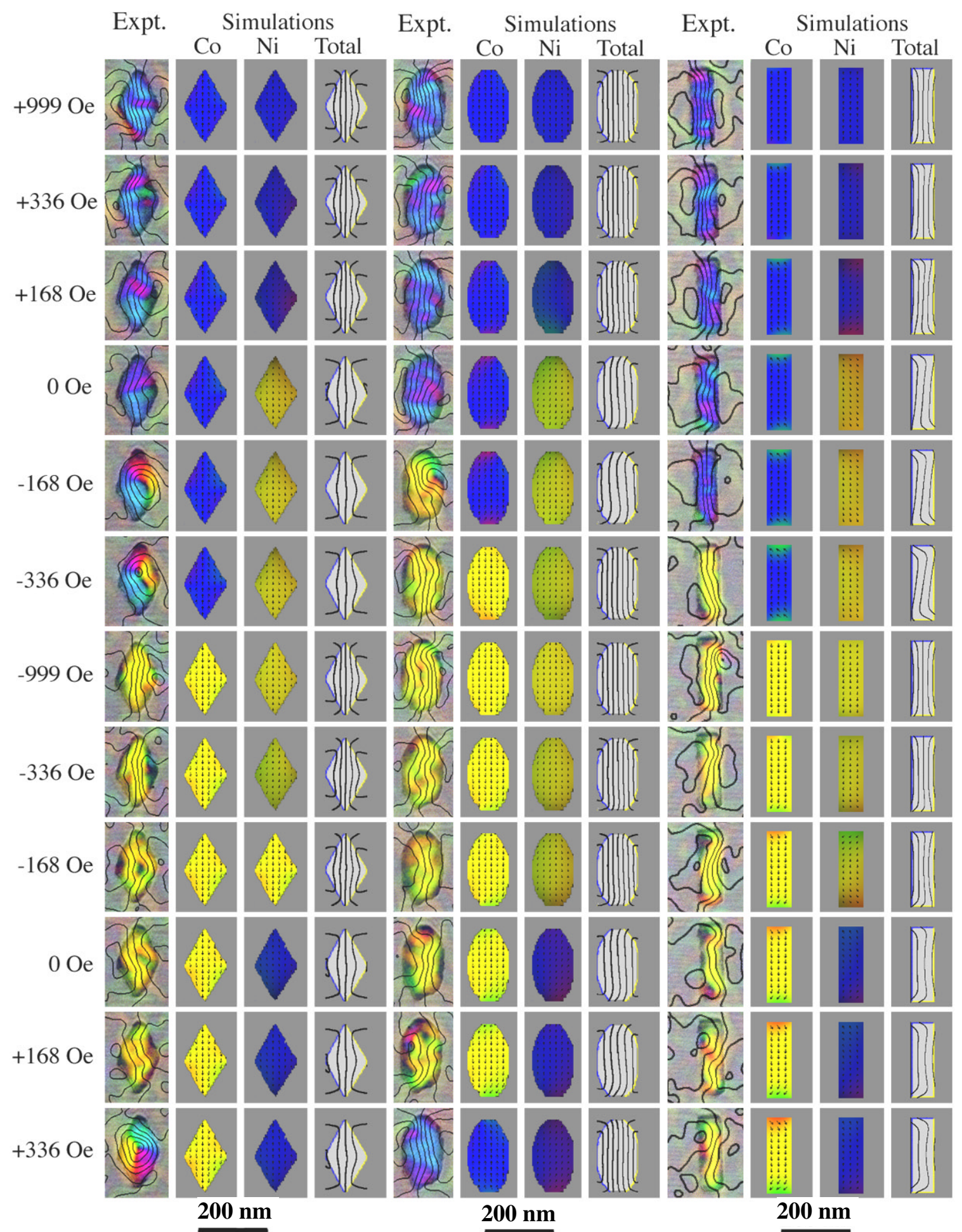

Fig.4. Experimental hysteresis cycles for second largest $\mathrm{Co} / \mathrm{Au} / \mathrm{Ni}$ diamonds, ellipses and bars in Fig. 3b, for average out-of-plane field of 3600 Oe and in-plane fields indicated applied along major axis of each shape. Contour spacing is $0.064 \pi$ radians. Simulations show magnetization in Co and Ni layers separately, as well as computed phase contours for direct comparison with data. 

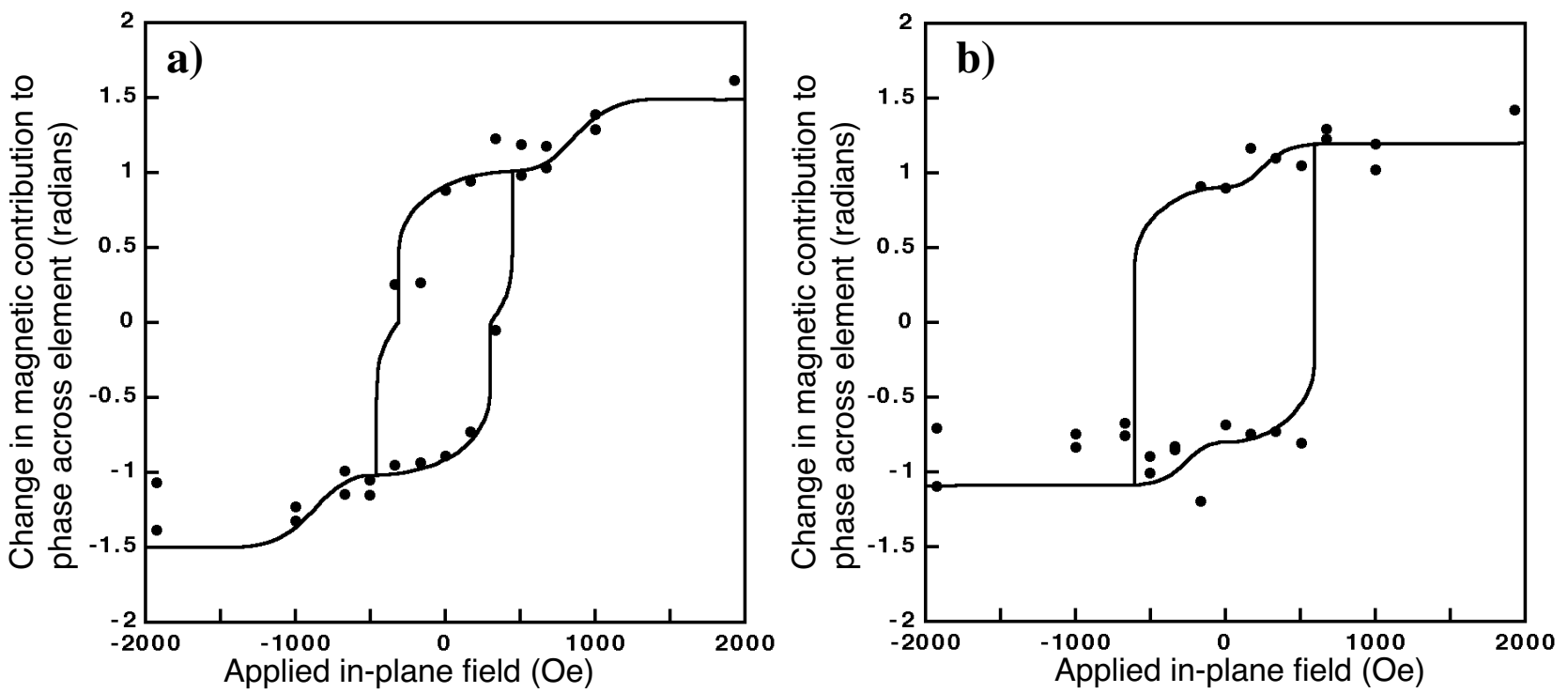

Fig.5. Hysteresis loops deduced from magnetic contributions to holographic phases of elements shown in Fig. 3 for a) largest diamonds and b) largest rectangular bars.

\section{CONCLUSIONS}

Magnetization reversal processes in submicron-sized magnetic elements have been followed using off-axis electron holography in the transmission electron microscope. Comparisons with micromagnetic simulations showed that the reproducibility of an element's domain structure in successive cycles is affected both by the out-of-plane component of the applied field and by the exact details of its starting magnetic state. Closely-separated magnetic layers within individual elements were observed to couple to each other antiferromagnetically, and narrow rectangular bars were found to reverse without the formation of end domains.

\section{ACKNOWLEDGMENTS}

We thank J. Speidell for the SiN membranes. The work was partly supported by an IBM subcontract on the DARPA Advanced MRAM Project (Contract No. MDA-972-96-C-0014).

\section{REFERENCES}

1. A. Tonomura, Ultramicroscopy 47, 419 (1992).

2. LLG Micromagnetics Simulator is available commercially. See www dancris.com/ $11 \mathrm{lg}$.

3. David J. Smith and M.R. McCartney, in Introduction to Electron Holography, edited by E. Volkl, L.F. Allard and D.C. Joy (Plenum, New York, 1998).

4. R.E. Dunin-Borkowski, M.R. McCartney, B. Kardynal and David J. Smith, J. Appl. Phys. 84, 374 (1998).

5. R.E. Dunin-Borkowski, M.R. McCartney, B. Kardynal, David J. Smith and M.R. Scheinfein, Appl. Phys. Lett. 75, 2641 (1999). 